\title{
TRAUMATIC DISLOCATION OF THE HIP IN CHILDHOOD
}

\author{
J. Piggot, Londonderry, Northern Ireland \\ From the Princess Elizabeth Orthopaedic Hospital, Exeter
}

According to Ambroise Paré, " there is this danger in the dislocation of the hip, that either the bone cannot be put into the place again, at least unless with much trouble, or else, being put in, that it will presently fall out again." These difficulties have been largely overcome. only to be replaced by the problem of avascular necrosis.

Mason (1954) reported one case of traumatic dislocation of the hip in a child, and found eighty-eight cases reported during the previous thirty-two years. Nine of these children later developed avascular necrosis of the affected femoral head-an incidence of 10 per cent. Several of the cases had not been adequately followed up, and, as Choyce (1924) concluded: " Had the earlier cases been followed up, the results would have been found to be less ideal than was supposed."
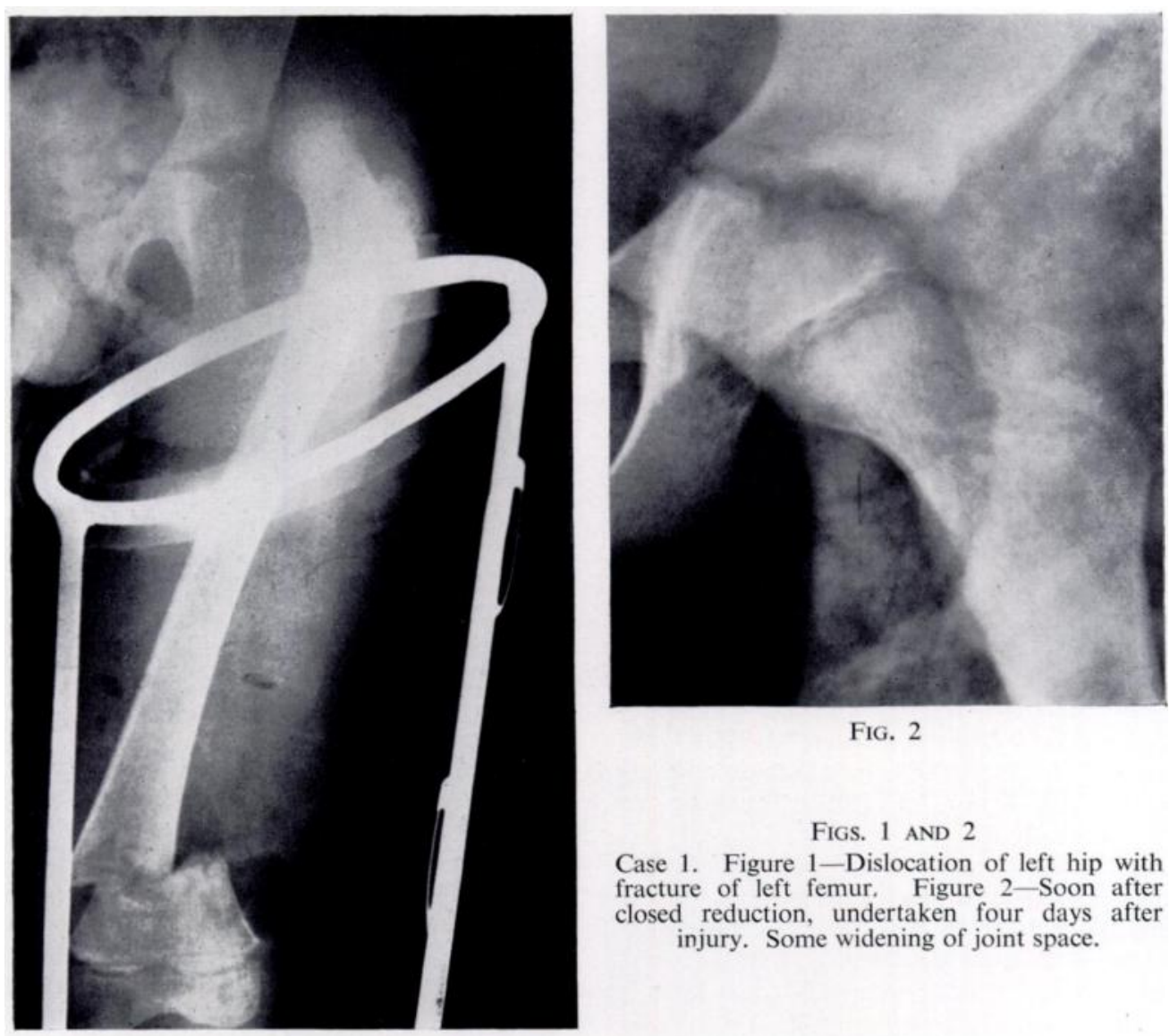

FIG. 2

Figs. 1 AND 2

Case 1. Figure 1-Dislocation of left hip with fracture of left femur. Figure 2-Soon after closed reduction, undertaken four days after injury. Some widening of joint space.

Fig. 1

During a recent review at Exeter of conditions of the hip nine cases of traumatic dislocation in children were encountered. Five were in boys and four in girls. In seven cases the dislocation was of the left hip, and in two of the right. The ages ranged from four and a half to fifteen 
years, the average being ten years. In all the epiphysial plate was clearly present at the time of injury. Avascular necrosis of the femoral head occurred in one case. The length of the periods of observation ranged from twenty-seven to 122 months, the average being sixty-four months.

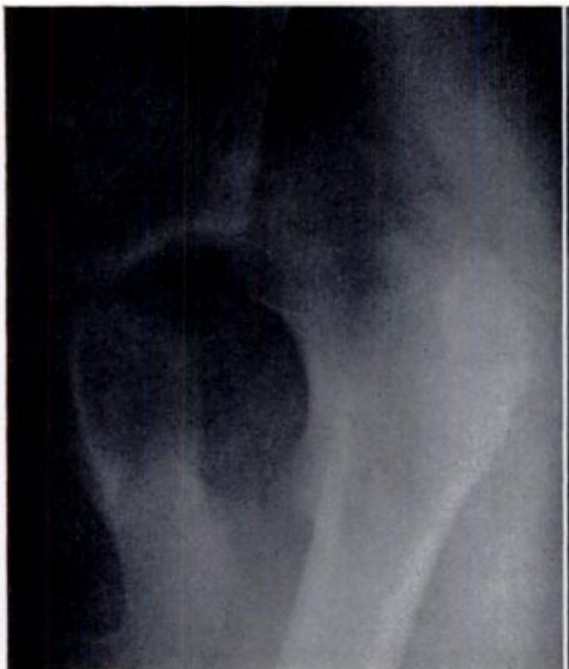

FIG. 3

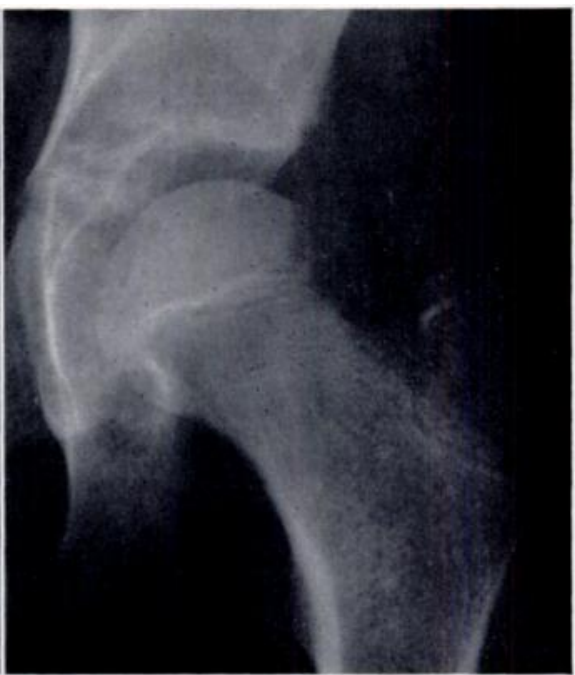

Fig. 4

Case 3. Figure 3-Dislocation of left hip. Figure 4-After six months the patient was free from symptoms, though the radiograph showed a relative increase in the density of the epiphysis.

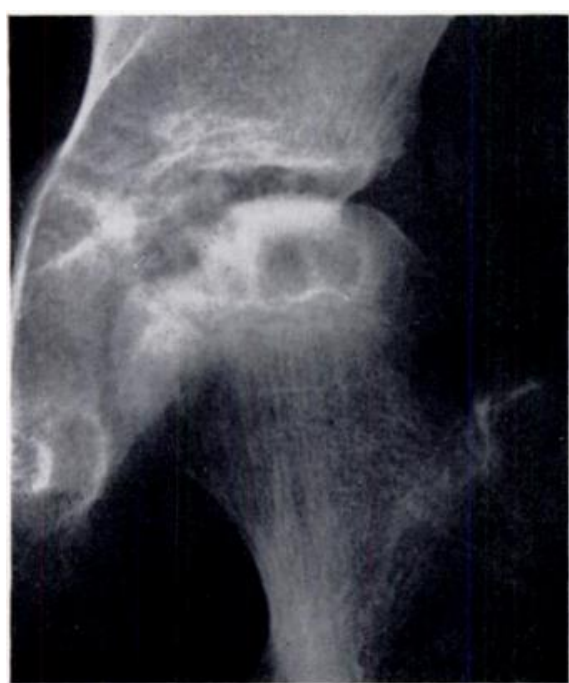

FIG. 5

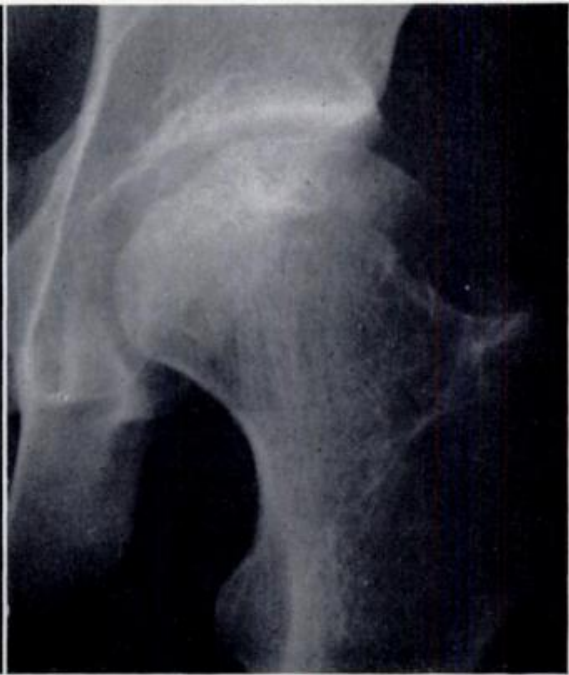

FIG. 6

Case 3. Figure 5-Cyst formation and some flattening of epiphysis. Figure 6-Seven years after injury. Symptom-free. Full range.

\section{CASE REPORTS}

Case 1-A boy of seven sustained multiple injuries in a fall from a Dodgem car in January 1948. These included, besides a dislocation of the left hip (Fig. 1), a fracture of the left femur, fractures of the lower ends of both tibiae and fibulae and division of the left popliteal vessels. There was a delay of four days before reduction of the dislocation of the hip, which was not diagnosed until the boy was transferred to the orthopaedic unit. Radiographs taken soon after reduction showed some widening of the joint (Fig. 2), which was not apparent in radiographs taken three weeks later. The boy

VOL. 43 B, NO. 1, FEBRUARY 1961 
lay in a plaster spica for eleven weeks and over the next four weeks was allowed to walk though not to bear weight on the left leg. After this he wore a weight-relieving caliper for six months. Ten years and two months after injury he had no symptoms, but had slight limitation of movement of the hip. Radiographs then showed no evidence of deformity of the femoral head nor of degenerative change. Case 2-A girl of seven fell from a tree in September 1949, sustaining a dislocation of the left hip with a marginal fracture of the acetabulum. After reduction she was in a plaster spica for twelve weeks, recumbent for the first four weeks and for the last eight walking with crutches without bearing weight on the affected leg. Eight years and seven months after injury she was free from symptoms but had slight limitation of movement of the hip.

Case 3-A girl of nine dislocated her left hip in April 1951 in a fall from a roof forty feet high (Fig. 3). The dislocation was reduced and a plaster spica was applied. After one week of recumbency she was allowed to walk without weight bearing on the affected side. The plaster was removed after seven weeks, when full weight bearing was allowed. Six months after the injury she had no symptoms, but radiographs showed some increased density of the head of the left femur (Fig. 4). One month later her mother noticed that she limped when tired. Subsequent radiographs showed cystic changes in, and slight collapse of the epiphysis (Fig. 5). She was fitted with a weight-relieving caliper and continued to wear it for four years, when the femoral head appeared radiologically to be of normal density. Seven years after injury she had no symptoms and had a full range of movement of the hip. Radiographs (Fig. 6) showed some flattening of the femoral head.

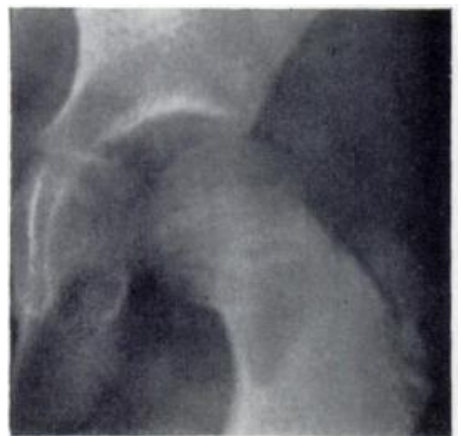

FIG. 7

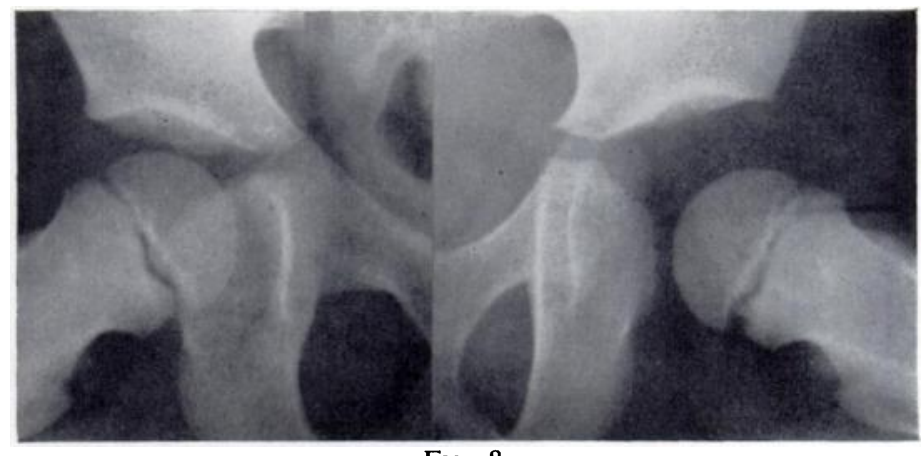

FIG. 8

Case 4. Figure 7-Dislocation of left hip. Figure 8-Marked widening of the joint after manipulative reduction.

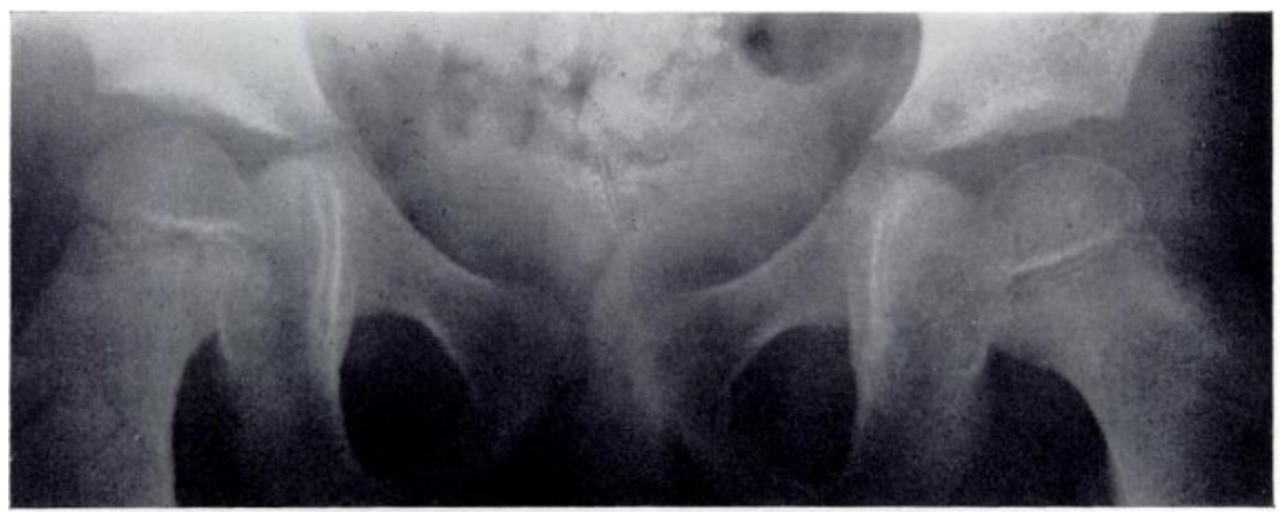

Fig. 9

Case 4. Figure 9-Eight weeks later. The joint space is now of equal width to that of the healthy side.

Case 4-A girl of four and a half was run over by a hay sweep in July 1951, sustaining a dislocation of the left hip (Fig. 7) and fractures of the left clavicle and right humerus. Radiographs taken after reduction showed a marked widening of the joint (Fig. 8). A plaster spica was applied, in which she was recumbent for eight weeks. After this full weight bearing was permitted. Radiographs taken eight weeks after reduction no longer showed the widening of the joint (Fig. 9). Six years and nine months after injury she was free from symptoms and had only slight limitation of movement of the hip. Radiographs showed no evidence of deformity of the femoral head nor of degenerative changes. 
Case 5-A boy of six in January 1954 crashed into a fence while tobogganing and dislocated his right hip. After reduction a plaster spica was applied in which he was recumbent for four weeks. The plaster was then removed and the boy walked with crutches, avoiding weight bearing for a further six weeks. Four years and one month later his hip was clinically normal.

Case 6-A boy of nine was knocked down by a car in January 1955, sustaining a dislocation of the right hip and a fracture of the skull. After reduction he was in a single plaster spica for ten weeks and bore no weight on the affected leg for fourteen weeks. Three years and one month after injury he had a full range of movement and was free from symptoms.

Case 7-A girl of fifteen fell off horizontal bars in February 1955, dislocating her left hip. After reduction the hip was immobilised in a plaster spica for eight weeks. Two weeks after injury she was allowed to walk with crutches, not bearing weight on the affected side for a total of thirteen weeks. Three years and two months after injury she had no symptoms from the hip. Radiographs were not taken because, being a student radiographer, the patient was averse to additional radiation.

Case 8-A boy of fifteen dislocated his left hip in October 1955 while playing football. After manipulative reduction the leg was kept on three pounds' skin traction for six weeks. During the next six weeks he walked with crutches, not bearing weight on the affected limb. Two years and eight months after injury he had no symptoms referable to the hip.

Case 9-A boy of fifteen was knocked down by a pedal cycle in December 1955 and sustained a dislocation of the left hip. After reduction, skin traction with three pounds' weight was maintained for five weeks. He was then in a plaster spica for twelve weeks and avoided bearing weight for seventeen weeks after injury. Two years and three months after injury he had no symptoms referable to the hip and had a full range of movement.

\section{DISCUSSION}

In Case 1 there was a delay in reducing the dislocation because the other injuries, particularly the fracture of the femur on the same side, distracted attention from the lesion of the hip.

Dehne and Immermann (1951) found that in forty-two cases of dislocation of the hip, with fracture of the femur on the same side, the dislocation was recognised at initial examination in only fifteen. In most of the remainder the diagnosis was not made until four to six months later, when failure of the femur to unite led to further investigation and the discovery of the unreduced dislocation. In the present case the hip was reduced quite easily in spite of the four days' delay. Some widening of joint space was seen in the radiograph taken soon after reduction.

More marked widening was apparent after reduction in Case 4. There was no delay in treatment as in Case 1, so that blood in the acetabulum should not have formed a firm clot. It is believed likely that soft-tissue interposition rather than haemarthrosis caused this widening of joint space. Paterson (1957) reported a similar case in which he undertook open reduction. The acetabular labrum was detached and lay across the joint. Something similar may have occurred in this case.

One patient developed avascular necrosis of the head of the femur. This girl must have sustained very considerable damage to her hip when she fell from a height of forty feet. She avoided movement and weight bearing on the affected side for seven seeks. At six months increased density of the head of the femur was noted, and one month later she was limping. The development of the radiographic appearances of avascularity before symptoms and signs has been described by Nicoll (1952) in a review of 144 adult cases.

The cause of avascular necrosis is generally thought to be tearing and thrombosis of vessels at the time of injury and reduction. Trueta (1957) has shown that the blood supply to the head of the femur during childhood differs from that during adult life, and that between the fourth year and puberty metaphysial vessels do not contribute to the circulation of the epiphysis.

The incidence of avascular necrosis in adults was reported by Nicoll (1952) to be 10 per cent. In his review of the literature Mason (1954) found a similar incidence in children. However, in comparing the incidence of avascular necrosis in childhood with that in adult life,

VOL. 43 B, NO. 1, FEBRUARY 1961 
the degree of violence required to produce the dislocation must be considered as well as differences in the vascular supply. When the bones of the hip are largely cartilaginous dislocation may be produced more easily than when they are fully ossified and less plastic.

In all cases the hip was immobilised after reduction either in a plaster spica or by light skin traction. Bearing weight on the affected limb was not allowed within seven weeks of injury. There is no evidence that the avoidance of weight bearing prevented avascular necrosis. Banks (1951), advocating avoidance of weight bearing for six months, claimed that the atrophy of disuse so caused showed up the relative increase in density of the head more distinctly, and so assisted the diagnosis of avascular necrosis. He did not claim that this treatment would prevent avascular necrosis.

\section{SUMMARY}

1. Nine cases of traumatic dislocation of the hip in children have been reviewed.

2. There was one instance of avascular necrosis of the femoral head, which became apparent six months after injury.

3. In one case fracture of the femur on the same side led to four days' delay in recognition and treatment of the dislocation.

4. At the time of review all patients were free from symptoms.

My thanks are due to Mr F. C. Durbin and Mr C. C. Jeffery for permitting me to review their cases, and to Mr Norman Capener for much help in the preparation of this paper.

\section{REFERENCES}

Banks, S. W. (1951): Traumatic Dislocation of the Hip. Discussion. Journal of Bone and Joint Surgery, 33-A, 777.

Choyce, C. C. (1924): Traumatic Dislocation of the Hip in Childhood, and Relation of Trauma to Pseudocoxalgia. British Journal of Surgery, 12, 52.

Clarke, H. Osmonde (1929): Traumatic Dislocation of the Hip Joint in a Child. British Journal of Surgery, 16, 690.

Dehne, E., and Immermann, E. W. (1951): Dislocation of the Hip Combined with Fracture of the Shaft of the Femur on the Same Side. Journal of Bone and Joint Surgery, 33-A, 731.

Mason, M. L. (1954): Traumatic Dislocation of the Hip in Childhood. Journal of Bone and Joint Surgery, 36-B, 630.

Nicoll, E. A. (1952): Traumatic Dislocation of the Hip Joint. Journal of Bone and Joint Surgery, 34-B, 503.

ParÉ, A. (1678): The Works of that Famous Chirurgeon, Ambrose Parey. Translated by Th. Johnson. London: J. Clark.

Paterson, I. (1957): The Torn Acetabular Labrum. Journal of Bone and Joint Surgery, 39-B, 306.

Thompson, V. P., and EPSTein, H. C. (1951): Traumatic Dislocation of the Hip. Journal of Bone and Joint Surgery, 33-A, 746.

Trueta, J. (1957): The Normal Vascular Anatomy of the Human Femoral Head During Growth. Journal of Bone and Joint Surgery, 39-B, 358. 